\title{
Risk factors for sleep-disordered breathing in pregnancy
}

\author{
Grace W Pien, 1,2,3 Allan I Pack, ${ }^{1,2}$ Nicholas Jackson, ${ }^{1}$ Greg Maislin, ${ }^{1}$ \\ George A Macones, ${ }^{4}$ Richard J Schwab ${ }^{1,2,3}$
}

\begin{abstract}
- Additional material is published online only. To view please visit the journal online (http://dx.doi.org/10.1136/ thoraxjn-2012-202718)

${ }^{1}$ Center for Sleep and Circadian Neurobiology, University of Pennsylvania School of Medicine, Philadelphia, Pennsylvania, USA

${ }^{2}$ Sleep Medicine Division, Department of Medicine, University of Pennsylvania School of Medicine, Philadelphia, Pennsylvania, USA

${ }^{3}$ Pulmonary and Critical Care Division, Department of Medicine, University of Pennsylvania School of Medicine, Philadelphia, Pennsylvania, USA

${ }^{4}$ Department of Obstetrics and Gynecology, Washington University School of Medicine, St Louis, Missouri, USA
\end{abstract}

\section{Correspondence to}

Dr Grace W Pien, Division of

Pulmonary and Critical Care Medicine, Johns Hopkins University School of Medicine, Johns Hopkins Allergy and Asthma Center, 5501 Hopkins Bayview Circle, Baltimore, MD 21224, USA gpien1@jhmi.edu

Received 11 September 2012 Revised 19 September 2013 Accepted 31 October 2013 Published Online First 21 November 2013

\begin{tabular}{l}
\hline To cite: Pien GW, Pack Al, \\
Jackson N, et al. Thorax \\
2014;69:371-377. \\
\hline
\end{tabular}

\section{ABSTRACT}

Rationale Symptoms of sleep-disordered breathing (SDB) are common among pregnant women, and several studies link SDB symptoms with gestational hypertension and preeclampsia. However, few prospective studies objectively measuring SDB during pregnancy have been performed.

Objectives We performed a prospective cohort study examining risk factors for third trimester SDB in pregnant women.

Measurements and methods 105 pregnant women from the Hospital of the University of Pennsylvania obstetrics practices completed first and third trimester overnight polysomnography studies. We examined whether the number of SDB events per hour of sleep increased during pregnancy. We performed unadjusted and multivariable logistic regression analyses to estimate the effects of usual and pregnancy-specific characteristics on development of third trimester obstructive sleep apnoea (OSA). In secondary analyses, we examined the relationship between objectively measured SDB, hypertensive disorders of pregnancy, and other adverse maternal-fetal outcomes.

Main results Mean Apnoea-Hypopnoea Index increased from 2.07 (SD 3.01) events/h at baseline (first trimester) to 3.74 (SD 5.97) in the third trimester ( $p=0.009$ ). $10.5 \%$ of women had OSA in the first trimester. By the third trimester, $26.7 \%$ of women had OSA. In multivariable analyses, first trimester body mass index (BMI) and maternal age were significantly associated with third trimester OSA.

Conclusions Third trimester OSA is common. Risk factors for third trimester OSA among women without baseline SDB include higher baseline BMI and maternal age.

\section{INTRODUCTION}

The prevalence of sleep-disordered breathing (SDB) is low among women of reproductive age compared with postmenopausal women and men. ${ }^{1}$ However, during pregnancy, physiologic and hormonal changes occur that can lead to a greater propensity for apneic episodes during sleep (reviewed in Ref. 2).

In initial case reports of obstructive sleep apnoea (OSA) in pregnancy, women were uniformly obese and often developed complications such as preeclampsia. ${ }^{3}{ }^{4}$ Subsequently, studies have demonstrated that snoring and other SDB symptoms are common among pregnant women and suggest that pregnancy may accelerate development of SDB. ${ }^{5-7}$ Nevertheless, data on whether objectively measured

\section{Key messages}

What is the key question?

- Do objectively measured sleep-disordered breathing (SDB) events increase during pregnancy and what are risk factors for gestational obstructive sleep apnoea (OSA)?

\section{What is the bottom line?}

- SDB events increase over the course of pregnancy and third trimester OSA is common. Age and Body Mass Index are significant and consistent predictors of gestational OSA.

\section{Why read on?}

- We report on risk factors for third trimester OSA in a cohort of pregnant women who underwent objective measurements of SDB in the first and third trimesters. In secondary analyses, we examine the relationships between SDB and maternal fetal outcomes. The findings have implications for identifying women at risk for OSA during pregnancy.

SDB increases during pregnancy and on risk factors for incident OSA in pregnant women are limited.

A number of studies have examined the relationship between SDB and gestational hypertension or preeclampsia, since OSA clearly increases hypertension risk in the general population. ${ }^{8}$ These studies largely suggest that SDB is a risk factor for preeclampsia, ${ }^{6}{ }^{9-14}$ though in most cases, their ability to infer causality has been limited by their crosssectional or retrospective nature, or reliance on self-reported symptoms of SDB. Few studies, to date, have assessed the impact of objectively measured gestational SDB on maternal-fetal health.

In this study, we performed a prospective cohort study to determine whether SDB events increased during pregnancy; and examined associations between weight, age and third trimester OSA. We hypothesised that greater baseline weight and gestational weight gain would increase the risk for OSA. In secondary analyses, we also investigated the relationship between SDB and gestational hypertension and preeclampsia; and between SDB and other adverse maternal-fetal outcomes, including preterm delivery, gestational diabetes (GDM) and low birthweight babies. 


\section{METHODS}

\section{Study population}

All women scheduled for initial obstetrics evaluation at the Hospital of the University of Pennsylvania were invited to participate. Interested women were screened for eligibility. This study was approved by the Penn Institutional Review Board. All participants provided written informed consent.

Women whose pregnancy was $\leq 14$ weeks of gestation were eligible. Gestational age and estimated date of delivery (EDD) were calculated using last menstrual period data. ${ }^{15}$ When available $(n=96)$, fetal ultrasonography results were used to confirm EDD. ${ }^{15}$ Exclusion criteria were communication/cognitive/behavioural impairments interfering with informed participation; no telephone; self-reported illicit drug use or alcoholism ${ }^{16}$; serious pre-existing medical conditions; sedative/hypnotic use $\geq 3 \times /$ week; or current OSA treatment.

To enrich the sample for subjects likely to develop OSA, recruitment was stratified by BMI to increase the proportion of obese women: normal $\left(<25.0 \mathrm{~kg} / \mathrm{m}^{2}\right)$, overweight $(25.0-$ $<30.0)$, class I obesity $(30.0-<35.0)$, class II-III obesity $(\geq 35.0) .{ }^{17}$ Our recruitment goal was 25 subjects/category; subjects who withdrew were replaced until the goal was reached.

In total, 722 interested women were screened for inclusion into the cohort, of whom 352 women met eligibility criteria. By far the most common reason for exclusion was pregnancy past 14 weeks of gestation $(n=325)$. Others were excluded due to not being pregnant/pregnancy loss/planned termination $(n=17)$; chronic medical conditions $(n=4)$; prenatal care at other institutions $(n=9)$; or lack of English fluency $(n=1)$. Many otherwise eligible women were not enrolled due to stratified recruitment by BMI $(n=172)$ or because they were unable to attend first trimester polysomnography (PSG) prior to 14 weeks of gestation $(n=54)$.

\section{Protocol}

We obtained medical, obstetric and sleep histories at baseline (first trimester) evaluation. Overnight laboratory PSG was scheduled between 8 and 14 weeks gestation, and for repeat between 33 and 34 weeks gestation. PSGs (Sandman, Melville Diagnostics) included EEG (central, occipital leads), electrooculogram, submental and tibialis electromyograms, single bipolar electrocardiogram, finger pulse oximetry (Nellcor), chest and abdominal excursion (Protech piezo belts), airflow by nasal pressure $^{18}$ and oral thermistor. Accepted methods were used to score sleep. ${ }^{19}$

We defined apnoea as complete cessation of airflow lasting $\geq 10 \mathrm{~s}$, and hypopnoea as a $>50 \%$ decrease in airflow without a requirement for associated oxygen desaturation or arousal; or a lesser airflow reduction in association with oxygen desaturation of $>3 \%$ or an arousal. ${ }^{20}$ The Apnoea-Hypopnoea Index (AHI, apnoea+hypopnoea/h of sleep) was computed using standard criteria. ${ }^{19}{ }^{20}$ Subjects with desaturations to $<70 \%$ for $\geq 3 \%$ of time asleep and/or AHI $>30$ were notified $(n=1)$ and withdrawn if they elected treatment $(n=1)$.

Before each PSG, the Epworth Sleepiness Scale ${ }^{21}$ and a seated manual blood pressure (BP) measurement were obtained. After delivery, charts were abstracted for maternal-fetal outcomes.

\section{Outcome variables}

We examined two primary SDB outcomes: first, AHI was dichotomised at $\geq 5$ events/h to define OSA in the primary analyses. We also examined change in $\mathrm{AHI}$ as a continuous variable.
Gestational hypertension was defined clinically as systolic BP $\geq 140 \mathrm{~mm} \mathrm{Hg}$ and/or diastolic BP $\geq 90$ in a previously normotensive woman $>20$ weeks gestation. Preeclampsia was defined as gestational hypertension with $24 \mathrm{~h}$ urinary protein level $>0.3 \mathrm{~g}$. We also examined third trimester mean arterial BP (calculated from BP obtained prior to third trimester PSG), preterm delivery (gestational age $<37.0$ weeks), GDM (defined per $^{22}$ ) and low birthweight babies $(<2500 \mathrm{~g})$ as secondary outcomes.

\section{Determinant variables}

Determinant variables included first and third trimester BMI and neck circumference, gestational weight gain, age, race, baseline AHI, Epworth Sleepiness Scale scores and parity. Weight and gestational weight gain were examined using several paradigms, such as categorised and continuous BMI, neck circumference and absolute weight change from baseline until delivery.

\section{Data analyses}

Data were analysed with SAS software (V.9.1, SAS Institute, Cary, North Carolina, USA) for descriptive statistics and multivariable logistic and linear regression. Because AHI distribution was substantially non-normal, a log transformation (log (AHI +1)) was employed for comparisons of AHI and when AHI was used in linear regression as an outcome. For analyses in which AHI was dichotomised and when we examined change in AHI (which was normally distributed) as an outcome, we used untransformed AHI.

The power calculation was based on our primary outcome of absolute change in AHI. We calculated 25 subjects completing both PSGs in each BMI group $(n=100)$ were needed to detect a standardised effect size of 0.28 with $80 \%$ power using a twosided paired t test and 5\% significance level. That is, if the mean AHI change is approximately one-fourth the size of the SD of changes, our study is likely to find that the mean difference is significantly different from 0 .

We restricted our initial analyses to subjects who completed both overnight PSG studies $(n=105)$. To maximise statistical power, analyses were repeated after imputing third trimester AHI for 21 women, using a regression model of baseline AHI, age, BMI, race, chronic hypertension, parity, gestational hypertension and preeclampsia. ${ }^{23}$

Paired $t$ tests were performed to compare first and third trimester log-transformed AHI, and characteristics of women with and without third trimester OSA. After estimating unadjusted associations of each variable with third trimester OSA, multivariable logistic regression models were used to estimate the effects of covariates on the outcome. Covariates were considered for inclusion in the final model if the $p$ value was $\leq 0.20$ in the unadjusted analyses. We also checked for collinearity between potentially related variables using Spearman's coefficient (eg, baseline BMI and gestational weight gain, baseline BMI and age, BMI and neck circumference) during the model selection process. Final covariates were selected based on whether each variable remained significant at $\mathrm{p}<0.05$ in the multivariable model. We also performed linear regression analyses to examine associations with change in AHI. Because AHI cut-point for OSA affects disease prevalence, we repeated our primary analyses using AHI $\geq 10$ events/h to verify our observations.

We performed logistic regression analyses using continuous and dichotomised SDB variables as independent variables to examine relationships between SDB and the hypertensive disorders of pregnancy, preterm delivery, GDM and low birth weight individually as outcomes. We also examined the relationship 
between SDB and third trimester mean arterial BP using linear regression. $\mathrm{p}$ Values $<0.05$ were considered statistically significant.

\section{RESULTS}

We enrolled 126 women in the cohort; 105 women completed first and third trimester PSGs at a median of 12.1 (SD 1.9) and 33.6 (SD 2.5) weeks gestation (table 1); 21 women completed first trimester PSGs only; $75 \%$ of participants were AfricanAmerican. Caucasian women and women of other racial backgrounds were combined into a 'White and Other' category. Reasons for failure to complete the third trimester PSG included delivery before the PSG $(n=6)$, preterm labour/medically advised bed rest $(n=6)$ and withdrawal/loss to follow-up $(n=9)$. Women delivered at a median of 39.1 (SD 2.0) weeks. Comparisons between women completing one and both PSGs revealed no significant differences in demographic, gynaecological or baseline sleep variables (including age, race, parity, baseline AHI). Some outcomes were unavailable for 12 subjects delivered at other hospitals.

\section{SDB increases during pregnancy}

Mean first trimester AHI was 2.07 (Med 1.1) events/h for subjects completing both PSGs. Third trimester AHI increased significantly to 3.74 (Med 1.5) events/h ( $p=0.009) ; 10.5 \%$ of women in our study had first trimester OSA (AHI $\geq 5)$. By the third trimester, $26.7 \%$ of our participants had OSA, with 23 mild (AHI 5-14), 4 moderate (AHI 15-29) and 1 severe $(\mathrm{AHI} \geq 30)$ case. $^{24}$

Women with third trimester OSA were mostly obese (BMI $\geq 30$ ) at baseline (table 2): 20 of 50 (40.0\%) obese women at baseline assessment had third trimester OSA, V.8 of 55 $(14.5 \%)$ normal or overweight women. Third trimester OSA was characterised largely by arousal-associated hypopnoeas. Women with third trimester OSA were not sleepier than normal women.

As our protocol over-recruited for overweight and obese women, we utilised data from all women who were screened in

Table 1 Characteristics of subjects completing first and third trimester polysomnography $(n=105)$

\begin{tabular}{lc}
\hline Characteristic & $\begin{array}{c}\text { Subjects completing } \\
\text { both PSGs }\end{array}$ \\
\hline Age, years (SD) & $26.7(7.2)$ \\
Race, $\mathrm{n}(\%)$ & \\
African-American & $79(75.2)$ \\
Caucasian & $22(21.0)$ \\
Other & $4(3.8)$ \\
Baseline BMI, kg/m² (SD) & $33.4(6.4)$ \\
Normal, $\mathrm{n}(\%)$ & $30(28.6)$ \\
Overweight, $\mathrm{n}(\%)$ & $25(23.8)$ \\
Class I obesity, $\mathrm{n}$ (\%) & $25(23.8)$ \\
Class II-III obesity, $\mathrm{n}(\%)$ & $25(23.8)$ \\
Gestational weight gain, kg (SD) & $8.9(5.5)$ \\
1st trimester neck circumference, cm (SD) & $35.7(3.0)$ \\
1st trimester AHI, events/hour (Median) & $2.07(1.1)$ \\
Nulliparous, $\mathrm{n}(\%)$ & $32(30.5)$ \\
Singleton gestation, $\mathrm{n}$ (\%) & $102(97)$ \\
Chronic hypertension, $\mathrm{n}$ (\%) & $5(4.76)$ \\
History of hypertensive disorder of pregnancy, $\mathrm{n}(\%)$ & $9(8.6)$ \\
\hline AHI, Apnoea-Hypopnoea Index; BMI, Body Mass Index; PSG, polysomnography.
\end{tabular}

Table 2 Characteristics of women with and without third trimester OSA

\begin{tabular}{|c|c|c|c|}
\hline & $\begin{array}{l}\text { Third trimester } \\
\text { OSA }(n=28)\end{array}$ & $\begin{array}{l}\text { No 3rd } \\
\text { trimester OSA } \\
(n=77)\end{array}$ & $\mathrm{p}$ Value \\
\hline Baseline BMI, kg/m² (SD) & $34.1(7.9)$ & $28.5(6.3)$ & 0.002 \\
\hline $\begin{array}{l}\text { Gestational weight gain, } \\
\mathrm{kg}(\mathrm{SD})\end{array}$ & $7.0(5.2)$ & $9.6(5.5)$ & 0.034 \\
\hline Third trimester $\mathrm{BMI}, \mathrm{kg} / \mathrm{m}^{2}$ (SD) & $37.0(7.4)$ & $32.1(5.5)$ & 0.003 \\
\hline $\begin{array}{l}\text { Third trimester Epworth } \\
\text { score (SD) }\end{array}$ & $10.6(4.1)$ & $9.2(4.0)$ & 0.129 \\
\hline $\begin{array}{l}\text { First trimester AHI, events/h } \\
\text { (SD, median) }\end{array}$ & $4.08(4.55,2.6)$ & $1.34(1.74,0.7)$ & \\
\hline $\begin{array}{l}\text { Third trimester AHI, events } / \mathrm{h} \\
\text { (SD, median) }\end{array}$ & $10.97(7.69,8.3)$ & $1.11(1.24,0.6)$ & \\
\hline Apnoea Index & $1.79(3.62)$ & $0.12(0.25)$ & \\
\hline Hypopnoeas with arousal & $7.30(5.26)$ & $0.84(1.08)$ & \\
\hline $\begin{array}{l}\text { Hypopnoeas with } 3 \% \\
\text { desaturation }\end{array}$ & $1.89(2.33)$ & $0.15(0.37)$ & \\
\hline
\end{tabular}

the first trimester and provided information about BMI $(n=335)$ to estimate OSA prevalence among the general obstetric population from which our subjects were recruited. From these data, we estimate overall OSA prevalence in the clinical population to be $8.4 \%$ (95\% CI $5.6 \%$ to $11.9 \%$ ) in the first trimester, and $19.7 \%$ (95\% CI $15.6 \%$ to $24.4 \%)$ in the third trimester.

\section{Third trimester OSA}

We examined whether specific clinical characteristics were significantly associated with third trimester OSA (table 3). Because women with AHI $\geq 5$ at baseline were likely to have third trimester OSA; we restricted these analyses to women with first trimester AHI <5 $(n=94)$. In unadjusted analyses, first or third trimester BMI, first or third trimester neck circumference, age and parity were all significant determinants of OSA $(p<0.05)$. Additional variables examined for inclusion in multivariable models included baseline AHI and gestational weight gain $(\mathrm{p} \leq 0.20)$.

We created separate multivariable models using first and third trimester weight covariates to better understand the relationship between weight and third trimester SDB. As BMI and neck circumference were highly collinear $(\rho=0.80)$, we retained BMI since $\mathrm{BMI}$ is routinely measured clinically and models including BMI had greater predictive value (ie, higher c-statistics).

Using first trimester characteristics, we found that first trimester BMI and maternal age were all significant determinants of third trimester OSA (table 4). In the fully adjusted model, with every BMI increase of $5 \mathrm{~kg} / \mathrm{m}^{2}$, subjects were $1.93(95 \%$ CI 1.19 to 3.12 ) times more likely to have third trimester OSA. With each 10-year increase in age, subjects were 3.24 (95\% CI 1.40 to 7.52 ) times more likely to have third trimester OSA. We included baseline AHI in the model to adjust for its influence on third trimester AHI, though it was not significantly associated with OSA.

In our dataset, baseline BMI and gestational weight gain were inversely correlated but not collinear $(\rho=-0.53)$. Including gestational weight gain in the model with baseline BMI, age and first trimester AHI suggested that heavier women who gained 
Table 3 Unadjusted associations between BMI, age, other variables and development of third trimester OSA ( $n=94)$

\begin{tabular}{|c|c|c|}
\hline & $\begin{array}{l}\text { OR for 3rd trimester } \\
\text { OSA }(95 \% \mathrm{Cl})\end{array}$ & p Value \\
\hline Baseline BMI, $5 \mathrm{~kg} / \mathrm{m}^{2}$ & 1.79 (1.17 to 2.74$)$ & 0.007 \\
\hline Third trimester BMI, $5 \mathrm{~kg} / \mathrm{m}^{2}$ & 1.97 (1.21 to 3.22$)$ & 0.007 \\
\hline Baseline BMI & & $0.070^{*}$ \\
\hline Normal & Ref & - \\
\hline Overweight & $2.60(0.43$ to 15.65$)$ & 0.297 \\
\hline Class I obesity & $3.82(0.66$ to 22.00$)$ & 0.133 \\
\hline Class II-III obesity & 8.67 (1.59 to 47.15$)$ & 0.013 \\
\hline Third trimester BMI & & $0.043 *$ \\
\hline Normal & Ref & - \\
\hline Overweight & $0.08(0.00$ to 1.75$)$ & 0.107 \\
\hline Class I obesity & $0.37(0.03$ to 4.90$)$ & 0.451 \\
\hline Class II-III obesity & $1.16(0.09$ to 14.29$)$ & 0.909 \\
\hline Gestational weight gain, $\mathrm{kg}$ & $0.94(0.58$ to 1.03$)$ & 0.195 \\
\hline First trimester neck circumference, $\mathrm{cm}$ & $1.20(1.00$ to 1.43$)$ & 0.049 \\
\hline Third trimester neck circumference, $\mathrm{cm}$ & 1.22 (1.01 to 1.47$)$ & 0.040 \\
\hline Age, 10 years & $2.92(1.38$ to 6.18$)$ & 0.005 \\
\hline Baseline AHI, events/h & 1.30 (0.91 to 1.86$)$ & 0.153 \\
\hline First trimester Epworth score & $1.03(0.92$ to 1.16$)$ & 0.554 \\
\hline Third trimester Epworth score & 1.07 (0.94 to 1.22$)$ & 0.283 \\
\hline Nulliparity (ref multiparity) & $0.67(0.24$ to 1.86$)$ & 0.443 \\
\hline Parity (total number of prior pregnancies) & 1.31 (1.04 to 1.67$)$ & 0.025 \\
\hline $\begin{array}{l}\text { White and other ethnicity (ref African- } \\
\text { American) }\end{array}$ & $0.48(0.13$ to 1.83$)$ & 0.283 \\
\hline \multicolumn{3}{|c|}{$\begin{array}{l}\text { The bold numbers are statistically significant at the }<0.05 \text { level. } \\
\text { *Omnibus p value for BMI group differences in the logistic regression model. Pairwise } \\
\text { comparisons for subgroups are reported in italics for individual BMI groups, compared } \\
\text { to normal BMI. } \\
\text { AHI, Apnoea-Hypopnoea Index; BMI, Body Mass Index; OSA, obstructive sleep } \\
\text { apnoea. }\end{array}$} \\
\hline
\end{tabular}

more weight were at greatest risk for OSA, but the interaction between BMI and gestational weight gain was not significant $(p=0.274)$. Age and baseline BMI were not collinear.

In a multivariable model incorporating third trimester BMI (table 4), third trimester BMI and age were highly significantly determinants of third trimester OSA, similar to the first trimester model.

When we repeated our analyses using imputed third trimester AHI values for those without third trimester PSGs, we observed essentially identical results (see online supplement). Furthermore, in multivariable analyses using AHI $\geq 10 / \mathrm{h}$, baseline or third trimester BMI continued to predict third trimester OSA (OR 1.85-3.01, $\mathrm{p}=0.025-0.049$ ).

\section{Change in AHI}

We examined characteristics associated with change in AHI in all subjects completing both PSGs $(n=105$, table 5). In unadjusted analyses, first and third trimester BMI and third trimester neck circumference were all significant determinants of change in AHI. Larger gestational weight gains were associated with smaller AHI changes in unadjusted analyses. In the final multivariable model, first trimester BMI and first trimester AHI were all independently associated with change in AHI. Maternal age was of borderline significance $(p=0.059)$. Each increase in baseline BMI of $5 \mathrm{~kg} / \mathrm{m}^{2}$ was associated with an increase in AHI of $1.43(0.70-2.16)$. Higher baseline AHIs were paradoxically associated with decreases in AHI in the third trimester $(p=0.022)$. Similar results were observed when all subjects were included using imputed third trimester AHI (see online supplement).

\section{SDB and hypertensive disorders of pregnancy}

Twenty women developed a hypertensive disorder of pregnancy (gestational hypertension, $\mathrm{n}=9$; preeclampsia, $\mathrm{n}=10$; both, $\mathrm{n}=1$ ), including one with chronic hypertension and preeclampsia (see online supplement). In unadjusted analyses restricted to subjects without chronic hypertension $(n=100$, table 6$)$, there were no significant associations between first or third trimester AHI, OSA or change in AHI and gestational hypertension or preeclampsia. Likewise, associations between first or third trimester SDB variables, and third trimester mean arterial BP were not significant.

\section{SDB and other adverse maternal-fetal outcomes}

We examined the relationship between SDB variables and additional outcomes (table 7$)$ including preterm delivery $(n=10)$, GDM $(n=5)$ and low birthweight babies $(n=9)$. There were no significant associations between any SDB variables and these outcomes.

\section{DISCUSSION}

There are several major findings from our data. First, SDB events increase during pregnancy. Second, milder OSA is a common third trimester finding, but severe OSA is uncommon. Third, age and BMI were consistent and independent determinants of third trimester OSA. Finally, in secondary analyses, OSA, assessed early or late in pregnancy, was not associated with development of gestational hypertension or preeclampsia in this sample, or with preterm delivery, GDM or low birth weight.

Aside from a small case-control study $(n=22),{ }^{25}$ our study is the first to objectively assess SDB prospectively in a large group of pregnant women. We found that AHI increased significantly during pregnancy. The overall magnitude of this increase was small, and mean AHI remained within normal. However, $10.5 \%$ of participants had OSA even in the first trimester. By the third trimester, $26.7 \%$ of women had mild OSA, and $4.8 \%$ had moderate-severe OSA. As our study over-recruited obese women, these participants do not represent a general obstetrics population. However, extrapolating to the BMI distribution of

Table 4 Multivariable models for development of third trimester OSA

\begin{tabular}{|c|c|c|c|c|}
\hline Variable & ORs using baseline $\mathrm{BMI}(95 \% \mathrm{CI})$ & p Value & ORs using third trimester $\mathrm{BMI}(95 \% \mathrm{Cl})$ & $\mathrm{p}$ Value \\
\hline Age, 10 years & 3.24 (1.40 to 7.52$)$ & 0.006 & 4.04 (1.62 to 10.05$)$ & 0.003 \\
\hline Baseline BMI, $5 \mathrm{~kg} / \mathrm{m}^{2}$ & $1.93(1.19$ to 3.12$)$ & 0.008 & & \\
\hline Third trimester BMI, $5 \mathrm{~kg} / \mathrm{m}^{2}$ & & & 2.36 (1.32 to 4.22$)$ & 0.004 \\
\hline Baseline $\mathrm{AHI}$ & $1.03(0.67$ to 1.57$)$ & 0.904 & $1.10(0.72$ to 1.71$)$ & 0.653 \\
\hline
\end{tabular}

The bold numbers are statistically significant at the $<0.05$ level.

AHI, Apnoea-Hypopnoea Index; BMI, Body Mass Index; OSA, obstructive sleep apnoea. 
Table 5 Unadjusted and adjusted relationships between BMI, age, other variables and change in AHI $(n=105)$

\begin{tabular}{|c|c|c|c|c|}
\hline Variable & Unadjusted change in $\mathrm{AHI}$, events/h $(95 \% \mathrm{Cl})$ & p Value & Adjusted change in $\mathrm{AHI}$, events $/ \mathrm{h}(95 \% \mathrm{Cl})$ & p Value \\
\hline Baseline BMI, $5 \mathrm{~kg} / \mathrm{m}^{2}$ & $1.11(0.43$ to 1.80$)$ & 0.002 & $1.43(0.70$ to 2.16$)$ & 0.0002 \\
\hline Third trimester BMl, $5 \mathrm{~kg} / \mathrm{m}^{2}$ & $1.13(0.34$ to 1.92$)$ & 0.016 & & \\
\hline Baseline BMI & & $0.070^{*}$ & & \\
\hline Normal & Ref & - & & \\
\hline Overweight & $1.76(-1.00$ to 4.52$)$ & 0.211 & & \\
\hline Obese I & $2.11(-0.65$ to 4.87$)$ & 0.134 & & \\
\hline Obese II/III & 3.77 (1.01 to 6.54$)$ & 0.007 & & \\
\hline Gestational weight gain $(\mathrm{kg})$ & $-0.23(-0.42$ to -0.05$)$ & 0.012 & & \\
\hline First trimester neck circumference, $\mathrm{cm}$ & $0.33(-0.00$ to 0.67$)$ & 0.052 & & \\
\hline Third trimester neck circumference, $\mathrm{cm}$ & $0.44(0.08$ to 0.80$)$ & 0.016 & & \\
\hline Age, 10 years & $1.03(-0.37$ to 2.44$)$ & 0.149 & $1.32(-0.05$ to 2.68$)$ & 0.059 \\
\hline Baseline AHI & $-0.09(-0.43$ to 0.25$)$ & 0.604 & $-0.42(-0.77$ to -0.06$)$ & 0.022 \\
\hline Third trimester ESS & $0.19(-0.07$ to 0.45$)$ & 0.150 & & \\
\hline Change in ESS & $0.02(-0.07$ to 0.37$)$ & 0.186 & & \\
\hline Nulliparity & $-0.01(-2.06$ to 2.03$)$ & 0.990 & & \\
\hline Total parity & $0.39(-0.12$ to 0.90$)$ & 0.134 & & \\
\hline White and other ethnicity (ref African-Am) & $-1.91(-4.25$ to 0.42$)$ & 0.108 & & \\
\hline
\end{tabular}

The bold numbers are statistically significant at the $<0.05$ level.

${ }^{*}$ Omnibus $p$ value for BMI group differences in the logistic regression model. Pairwise comparisons for subgroups are reported in italics for individual BMI groups, compared with normal BMI.

AHI, Apnoea-Hypopnoea Index; BMI, Body Mass Index.

all women screened for the study, we estimate nearly one out of five of women in our obstetrics practices may have third trimester OSA, with 3\% developing severe OSA.

We identified several risk factors for third trimester OSA. Models using first and third trimester characteristics were ultimately very similar. Increasing first or third trimester BMI and age were strong and independent determinants of OSA. We obtained similar results when examining change in AHI, utilising imputed data for subjects with missing third trimester AHIs and using a higher AHI cutoff for OSA. Thus, we conclude that

Table 6 Unadjusted associations of SDB and other variables with hypertensive disorders of pregnancy $(n=100)$

\begin{tabular}{lll}
\hline Variable & $\begin{array}{l}\text { OR for pregnancy-induced } \\
\text { hypertension }(95 \% \mathrm{Cl})\end{array}$ & p Value \\
\hline 1st trimester AHI & $1.04(0.90$ to 1.21$)$ & 0.584 \\
3rd trimester AHI & $0.96(0.86$ to 1.07$)$ & 0.485 \\
1st trimester OSA (AHI $\geq 5$ events/h) & $0.44(0.05$ to 3.74$)$ & 0.455 \\
3rd trimester OSA (AHI $\geq 5$ events/h) & $0.50(0.13$ to 1.90$)$ & 0.310 \\
Change in AHI & $0.93(0.82$ to 1.06$)$ & 0.270 \\
Age, 10 years & $0.52(0.23$ to 1.16$)$ & 0.109 \\
1st trimester BMI, 5 unit & $1.17(0.84$ to 1.64$)$ & 0.348 \\
3rd trimester BMI, 5 unit & $1.27(0.87$ to 1.84$)$ & 0.220 \\
Gestational weight gain & $1.05(0.95$ to 1.15$)$ & 0.347 \\
1st trimester neck circumference & $1.13(0.96$ to 1.33$)$ & 0.135 \\
3rd trimester neck circumference & $1.10(0.94$ to 1.29$)$ & 0.235 \\
Nulliparity (ref=multi) & $3.00(1.03$ to 8.67$)$ & 0.043 \\
Prior history of hypertensive disorder & $1.29(0.24$ to 6.99$)$ & 0.765 \\
of pregnancy & & 0.883 \\
White and other ethnicity & $1.09(0.35$ to 3.40) & \\
(ref African-American) & & \\
\hline The bold numbers are statistically significant at the <0.05 level. & \\
AHI, Apnoea-Hypopnoea Index; BMI, Body Mass Index; 0SA, obstructive sleep \\
apnoea; SDB, sleep-disordered breathing.
\end{tabular}

risk factors for OSA in pregnant women are similar to those in the general population. ${ }^{8} 26$

We were surprised to find gestational weight gain was not independently associated with third trimester OSA, and that in unadjusted analyses, greater weight gain appeared protective against developing OSA. The inverse correlation between baseline BMI and gestational weight gain potentially provides an explanation, that is, normal weight women tended to gain more weight than overweight and obese women (similar to large observational studies ${ }^{27}$ ). In multivariable analyses, gestational weight gain did not significantly modify OSA risk among women of different baseline BMIs. However, this may have been due to inadequate statistical power.

Although OSA is usually characterised by excessive somnolence, the Epworth Sleepiness Scale score was not significantly associated with third trimester OSA or change in AHI in our study. This may be due to the general effects of hormonal and physical changes of pregnancy on sleep and sleepiness (eg, high levels of progesterone; physical discomfort contributing to sleep fragmentation). ${ }^{2}$ In previous work, we found the prevalence of self-reported excessive daytime sleepiness (ESS score $>10$ ) to be $31.0-45.5 \%$ over the course of pregnancy in a cohort of healthy women. ${ }^{5}$ Thus, during pregnancy, the presence of daytime somnolence may not reliably signal the presence of a clinically meaningful sleep disorder.

This is the first study to examine the relationship between objectively measured SDB and pregnancy-induced hypertension, an area of considerable interest in a prospective cohort study. We did not observe associations between various metrics for SDB and gestational hypertension or preeclampsia. By contrast, the prevalence of objective SDB in a recent case-control study was much higher among women with gestational hypertension compared with healthy pregnant controls, ${ }^{13}$ though the association between SDB and gestational hypertension was not significant after adjusting for BMI. Such findings raise questions about why disparities exist between our results and other retrospective or cross-sectional studies. ${ }^{928} 29$ There may be several reasons. 
Table 7 Unadjusted associations of SDB and other variables with gestational diabetes, preterm delivery and low birth weight

\begin{tabular}{|c|c|c|c|c|c|c|}
\hline & \multicolumn{2}{|c|}{ Gestational diabetes $(n=5)$} & \multicolumn{2}{|c|}{ Preterm delivery $(n=10)$} & \multicolumn{2}{|c|}{ Low birth weight $(n=9)$} \\
\hline & OR $(95 \% \mathrm{Cl})$ & $\mathrm{p}$ Value & OR $(95 \% \mathrm{Cl})$ & $\mathrm{p}$ Value & OR $(95 \% \mathrm{Cl})$ & p Value \\
\hline 1st TM AHI & 1.05 (0.82 to 1.34$)$ & 0.710 & 1.02 (0.84 to 1.24$)$ & 0.851 & $0.95(0.72$ to 1.25$)$ & 0.715 \\
\hline 3rd TM AHI & 1.03 (0.93 to 1.15$)$ & 0.536 & 1.02 (0.94 to 1.12$)$ & 0.586 & 1.03 (0.94 to 1.13$)$ & 0.546 \\
\hline 1st TM OSA & 2.39 (0.24 to 23.74$)$ & 0.457 & & & & \\
\hline 3rd TM OSA & 2.09 (0.33 to 13.27$)$ & 0.436 & 0.75 (0.15 to 3.84$)$ & 0.730 & 1.67 (0.38 to 7.34$)$ & 0.500 \\
\hline Change in $\mathrm{AHI}$ & 1.03 (0.91 to 1.18$)$ & 0.619 & $1.03(0.93$ to 1.14$)$ & 0.619 & $1.04(0.95$ to 1.15$)$ & 0.391 \\
\hline
\end{tabular}

First, we recognise that our study likely lacked adequate statistical power to detect an association with gestational hypertension and preeclampsia, especially given the relatively few participants with moderate or severe third trimester OSA $(n=5)$. Next, most of our subjects did not manifest OSA until the third trimester. Recently, a study using a national healthcare database demonstrated that risks for preeclampsia were higher among women with known antepartum OSA compared to age-matched controls. ${ }^{14}$ Thus, pre-existing OSA may represent a hazard for adverse outcomes during pregnancy, compared to incident gestational OSA. Finally, although the upper airway is smaller among preeclamptic women compared with normal pregnant women, ${ }^{12}$ objective assessments of SDB have generally been performed after identifying gestational hypertension or preeclampsia rather than beforehand. ${ }^{6} 92829$ Thus, whether SDB follows or precedes development of preeclampsia has not been established.

Recent data have suggested that pregnant women with SDB symptoms are at increased risk for other adverse maternal-fetal outcomes, especially GDM. ${ }^{30}{ }^{31}$ Among pregnant women with previously diagnosed OSA, GDM risk was increased even after adjusting for obesity. ${ }^{14}$ We did not observe an increased risk for GDM among women with SDB in our study. Since few of our subjects developed GDM, however, we lacked statistical power to make this determination. Additional studies to determine whether incident OSA in pregnancy increases GDM risk are needed to better understand this relationship.

Some other strengths and limitations of our study warrant mention. Our subjects were mostly urban, African-American women, who are traditionally under-represented in research studies but have high rates of $\mathrm{SDB},{ }^{8}$ gestational hypertension and preeclampsia. ${ }^{32}$ While race was not significantly associated with OSA in our study, our results may not be generalisable to other populations.

As third trimester PSG data were not available for 21 women, we imputed missing AHIs using accepted statistical methods. ${ }^{23}$ Baseline characteristics and results using imputed data and complete cases were similar, suggesting minimal selection bias among those completing both PSGs.

Our study provides new insights into SDB among pregnant women, demonstrating that mild third trimester OSA is common; and establishing that greater baseline BMI and maternal age increase the risk for third trimester OSA. By contrast with recent findings demonstrating increased risks of adverse maternal-fetal outcomes among women with known antepartum OSA, ${ }^{14}$ we did not observe associations between OSA and subsequent development of gestational hypertension or preeclampsia, or between OSA and preterm delivery, GDM or low birth weight in this longitudinal study. Larger, adequately powered studies that examine the impact of objectively measured SDB of different severities, including incident versus pre-existing OSA on pregnancy, are needed. Of immediate clinical relevance, our findings can help obstetricians to identify women at risk for OSA during pregnancy.

Acknowledgements We thank Elizabeth A Beothy and Bethany A Staley for their assistance in the conduct of the study, and the study participants, without whom this research would not have been possible.

Contributors GWP, AIP, RJS and GM conceived and designed the study. GWP also implemented the study, monitored data collection, and drafted the paper. She is guarantor. GM wrote the statistical analysis plan. NJ cleaned the data, ran the statistical analyses and revised the paper. GWP, AIP, NJ, GM and RJS interpreted results and revised the paper. GAM contributed to study design and revised the paper.

Funding This study was supported by grants from the National Institutes of Health (K23HD41465 and P01HL94307) and American Heart Association (0230190N).

Competing interests Financial support for the submitted work from National Institutes of Health and American Heart Association. GWP received an honorarium from the American Thoracic Society for an educational presentation and royalties for developing educational material for UpToDate.com. AIP has received royalties from McGraw Hill and funding from the Phillips Respironics Foundation for the John Miclot Professor endowment. GM is Principal Biostatistician of Biomedical Statistical Consulting (BSC), a CRO primarily involved in medical device regulatory trials. There is no overlap between this study and types of studies supported by BSC.

Patient consent Obtained.

Ethics approval University of Pennsylvania Institutional Review Board.

Provenance and peer review Not commissioned; externally peer reviewed.

\section{REFERENCES}

1 Bixler EO, Vgontzas AN, Lin HM, et al. Prevalence of sleep-disordered breathing in women: effects of gender. Am J Respir Crit Care Med 2001;163(3 Pt 1):608-13.

2 Pien GW, Schwab RJ. Sleep disorders during pregnancy. Sleep 2004;27:1405-17.

3 Charbonneau M, Falcone T, Cosio MG, et al. Obstructive sleep apnea during pregnancy. Therapy and implications for fetal health. Am Rev Respir Dis 1991; 144:461-3.

4 Kowall J, Clark G, Nino-Murcia G, et al. Precipitation of obstructive sleep apnea during pregnancy. Obstet Gynecol 1989;74(3 Pt 2):453-5.

5 Pien GW, Fife D, Pack Al, et al. Changes in symptoms of sleep-disordered breathing during pregnancy. Sleep 2005;28:1299-305.

6 Franklin KA, Holmgren PA, Jonsson F, et al. Snoring, pregnancy-induced hypertension, and growth retardation of the fetus. Chest 2000;117:137-41.

7 Loube DI, Poceta JS, Morales MC, et al. Self-reported snoring in pregnancy. Association with fetal outcome. Chest 1996;109:885-9.

8 Young T, Peppard PE, Gottlieb DJ. Epidemiology of obstructive sleep apnea: a population health perspective. Am J Respir Crit Care Med 2002;165:1217-39.

9 Bourjeily G, Raker CA, Chalhoub M, et al. Pregnancy and fetal outcomes of symptoms of sleep-disordered breathing. Eur Respir J 2010;36:849-55.

10 Connolly G, Razak AR, Hayanga A, et al. Inspiratory flow limitation during sleep in pre-eclampsia: comparison with normal pregnant and nonpregnant women. Eur Respir J 2001;18:672-6.

11 Edwards N, Blyton DM, Kirjavainen T, et al. Nasal continuous positive airway pressure reduces sleep-induced blood pressure increments in preeclampsia. Am J Respir Crit Care Med 2000;162:252-7

12 Izci B, Riha RL, Martin SE, et al. The upper airway in pregnancy and pre-eclampsia. Am J Respir Crit Care Med 2003;167:137-40.

13 Reid J, Skomro R, Cotton D, et al. Pregnant women with gestational hypertension may have a high frequency of sleep disordered breathing. Sleep 2011;34:1033-8. 
14 Chen $\mathrm{Y}-\mathrm{H}$, Kang J-H, Lin C-C, et al. Obstructive sleep apnea and the risk of adverse pregnancy outcomes. Am J Obstet Gynecol 2012;206:136.e1-5.

15 Mercer BM, Goldenberg RL, Meis PJ, et al. The Preterm Prediction Study: prediction of preterm premature rupture of membranes through clinical findings and ancillary testing. The National Institute of Child Health and Human Development Maternal-Fetal Medicine Units Network. Am J Obstet Gynecol 2000;183:738-45.

16 Ewing JA, Ewing JA. Screening for alcoholism using CAGE. Cut down, Annoyed, Guilty, Eye opener. [Erratum appears in JAMA 1999;281:611]. JAMA 1998;280:1904-5.

17 Flegal KM, Carroll MD, Kuczmarski RJ, et al. Overweight and obesity in the United States: prevalence and trends, 1960-1994. Int I Obes Relat Metab Disord 1998:22:39-47.

18 Montserrat JM, Farre R, Ballester E, et al. Evaluation of nasal prongs for estimating nasal flow. Am J Respir Crit Care Med 1997:155:211-15.

19 Rechstaffen A, Kales A. A manual of standardized terminology: techniques of scoring system for sleep states of human subjects. Brain Information Services/Brain Research Institute, UCLA, 1968.

20 Anonymous. Sleep-related breathing disorders in adults: recommendations for syndrome definition and measurement techniques in clinical research. The Report of an American Academy of Sleep Medicine Task Force. Sleep 1999;22:667-89.

21 Johns MW. A new method for measuring daytime sleepiness: the Epworth sleepiness scale. Sleep 1991;14:540-5.

22 Metzger BE, Coustan DR. Summary and recommendations of the Fourth International Workshop-Conference on Gestational Diabetes Mellitus. The Organizing Committee. Diabetes Care 1998;21(Suppl 2):B161-7.
23 Rubin DB, Schenker N. Multiple imputation in health-care databases: an overview and some applications. Stat Med 1991;10:585-98.

24 AASM. The International Classification of Sleep Disorders: Diagnostic and Coding Manual. 2nd edn. Westchester, IL: American Academy of Sleep Medicine, 2005.

25 Maasilta P, Bachour A, Teramo K, et al. Sleep-related disordered breathing during pregnancy in obese women. Chest 2001;120:1448-54.

26 Stradling JR, Crosby JH, Stradling JR, et al. Predictors and prevalence of obstructive sleep apnoea and snoring in 1001 middle aged men. Thorax 1991:46:85-90.

27 Chu SY, Callaghan WM, Bish CL, et al. Gestational weight gain by body mass index among US women delivering live births, 2004-2005: fueling future obesity. Am J Obstet Gynecol 2009;200:271.e1-7.

28 Champagne K, Schwartzman K, Opatrny L, et al. Obstructive sleep apnoea and its association with gestational hypertension. Eur Respir J 2009;33:559-65.

29 Louis JM, Auckley D, Sokol RJ, et al. Maternal and neonatal morbidities associated with obstructive sleep apnea complicating pregnancy. Am J Obstet Gynecol 2010;202:261.e1-5

30 Facco FL, Grobman WA, Kramer J, et al. Self-reported short sleep duration and frequent snoring in pregnancy: impact on glucose metabolism. Am J Obstet Gynecol 2010;203:142.e1-142.e5.

31 Qiu C, Enquobahri D, Frederick IO, et al. Glucose intolerance and gestational diabetes risk in relation to sleep duration and snoring during pregnancy: a pilot study. BMC Women's Health 2010:17. doi: 10.1186/1472-6874-10-17

32 Shen JJ, Tymkow C, MacMullen N, et al. Disparities in maternal outcomes among four ethnic populations. Ethn Dis 2005:15:492-7. 